\title{
miR-216b Downregulates Intestinal IL2RB to Inhibit the Invasion of Echinococcus granulosus Eggs in the Kazakh Sheep
}

\author{
Xuhai Wang ${ }^{1,2}$, Xin $\mathrm{Li}^{1}$, Fangyuan Yuan ${ }^{1}$, Chaocheng $\mathrm{Li}^{1}$, Bin Jia ${ }^{1 *}$ and Song Jiang ${ }^{1 *}$ \\ ${ }^{1}$ College of Animal Science and Technology, Shihezi University, Road Beisi, Shihezi \\ 832003, Xinjiang, P. R. China. \\ ${ }^{2}$ Chongqing Mintai New Agrotech Development Group Co., Ltd. Mintai road, shilong \\ industrial park, jiguanshi, Nanan district 400063, Chongqing. P. R. China.
}

\begin{tabular}{l} 
Article Information \\
Received 02 January 2020 \\
Revised 14 May 2020 \\
Accepted 02 June 2020 \\
Available online 15 June 2021 \\
Authors' Contribution \\
\hline $\mathrm{XW}$, XL and BJ designed the study \\
and wrote the manuscript. FY, CCL \\
and SJ performed experiments and \\
analyzed the data. \\
Key words \\
microRNAs, Molecular immunity, \\
Intestine tissue, Sheep, Cystic \\
echinococcosis
\end{tabular}

\begin{abstract}
A B S T R A C T
To study the early molecular immunity of Echinococcus granulosus infection in livestock, especially in sheep, the immunohistochemistry, in-situ hybridization was used to study the relationship and the distribution between miR-216b and IL2RB in the intestine tissue of kazakh sheep with resistant and non-resistant MHC haplotypes after oral infection with E. granulosus eggs. The results demonstrated that miR-216b negatively regulated IL2RB in the cystic echinococcosis intestinal tissues, and they were differentially expressed in the intestine tissue between the resistant sheep and non-resistant sheep, more widely distributed in the resistant group. These results illustrated that miR-216b and IL2RB activated stronger immune response in the resistant sheep, compared with the non-resistant sheep, and participating in the $\mathrm{T}$ cell immune response.
\end{abstract}

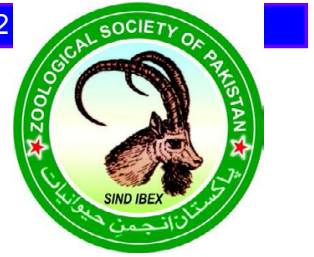

\section{INTRODUCTION}

$\mathrm{C}$ ystic echinococcosis (CE) is a complex, chronic and difficult to manage, a globally distributed zoonotic parasitic disease, the liver is the most important place for the presence of the parasitic cyst, which life cycle has three stages, includes adult, eggs and larvae (Jindra et al., 2010). Dogs and other canines are its definitive hosts. After the spores are eaten by the dog and then transported to its liver for colonization, in which they will develop into adults or ovulates and finally form cysts in livers of cattle or sheep. The health of people and livestock caused a great threat, and seriously affect the development of animal husbandry, resulting in significant economic losses.

Currently, the main methods of CE treatment include drug administration and surgery, which generally involves the administration of benzimidazole such as albendazole and mebendazole (Zhu et al., 2016). The shortcomings of these methods is that they are expensive and particularly challenging in underdeveloped countries and remote areas. Therefore, it is necessary to develop a relatively cheap and efficient treatment at the same time. Livestock vaccination may provide an additional method for $\mathrm{CE}$ management. Studies have been reported for the protection of certain

\footnotetext{
Corresponding author: jiabin@shzu.edu.cn; jiangsong918@shzu.edu.cn 0030-9923/2021/0005-1641 \$ 9.00/0

Copyright 2021 Zoological Society of Pakistan
}

animals, such as cattle, goats and sheep, because of Echinococcus granulosus cyst caused by CE (Larrieu et al., 2013). Hydatid disease infection can induce the production of Th1 and Th2 cytokines. Th1 cytokines are associated with protective immunity of echinococcosis, however Th2 cytokines can induce susceptible diseases. A large number of cell-mediated immune responses were found in the early stages of hydatidosis (Hui et al., 2015). However, the specific molecular immune mechanism of early infection is still unclear.

In our previous study, we examined the expression of differential genes of the sheep intestine tissue in response to the primary E. granulosus infection during the $4 \mathrm{~h}$ infection and produced extensive gene expression in the sheep. Such as IL17RE, IL1RL1, IL1R2, IL13, IL2RB are up-regulated, and these genes are associated with $\mathrm{T}$ cells activation (Magambo et al., 1995). Furthermore, microRNAs profile was conducted in the intestinal tissue of sheep with resistant and non-resistant $\mathrm{MHC}$ haplotypes after peroral infection with E. granulosus eggs, and the expression of a mass of microRNAs were significantly differentially, including up-regulated microRNAs (miR-101, miR-214, miR-182, miR-181, miR-184 etc.) and down-regulated microRNAs (miR-98, miR-128, miR-216b, miR-301 etc.), which are connected with the activation of $\mathrm{T}$ cells. microRNAs are important for the development and function of $\mathrm{T}$ cells, such as miR-101, miR-155, miR-214, miR-27 and miR31 , which are involved in the activation of $\mathrm{T}$ cells (Jindra 
et al., 2010; Magambo et al., 1995; Chiang et al., 2012; Fan et al., 2012; Glasmacher et al., 2010; Chen et al., 2012; Yu et al., 2007; Weitzel et al., 2009). To explore the molecular immunity mechanism of E. granulosus infection in livestock, ISH, IHC were used to analyze the relationship between differencial gene expression and miRNAs in a sheep model.

\section{MATERIALS AND METHODS}

\section{Sheep intestinal tissue}

All animals were in strict accordance with the the Animal Ethics Procedures and Guidelines of the People's Republic of China preparation and processing. The protocol was ratified by the Institutional Animal Protection Committee of Shihezi University. One resistanse healthy kazakh sheep (RG) and a non-resistanse healthy kazakh sheep (NRG as control) were kept in parasite-free conditions, and divided into resistance and non-resistance group. All of sheep were negative for antibodies to hydatid cyst fluid (HCF) antigen, carried out as previously reported (Hui et al., 2015). E. granulosus eggs were obtained from the gut content of three highly-infected dogs according to the method described (Veit et al., 1995). Handling the eggs were followed by Strict safety procedures (Jiang et al., 2016). Each sheep individual was infected perorally via a medical syringe with 8000 E. granulosus eggs suspended in $5 \mathrm{~mL}$ physiological saline. At $8 \mathrm{~h}$ postinfection, animals were sacrificed as previously reported (Glasmacher et al., 2010). At the same time, about $1 \mathrm{~cm}^{3}$ size of the intestinal tissue block were collected and fixed in the formalin prior to the immunohistochemistry (IHC) or in situ hybridization (ISH).

\section{Immunohistochemistry (IHC)}

The small intestine tissue of sheep from RG and NRG was fixed in 4\% formaldehyde. The small intestine tissue was embedded in paraffin and cut into a thickness of $5 \mu \mathrm{m}$. The slides were dried at $65{ }^{\circ} \mathrm{C}$ for $7 \mathrm{~h}$. The sections were dewaxed in Xylenes I and II for 5 min each and the sections were dehydrated with $100 \%, 95 \%, 90 \%$, and $80 \%$ ethyl alcohol for 2 min one time each, and then washed with distilled water (twice, 5 min each wash) and $3 \% \mathrm{H}_{2} \mathrm{O}_{2}$ to inactivate endogenous peroxidases (once, $5 \mathrm{~min}$ ). Antigen retrieval was carried out in $20 \times$ Tris EDTA ( $\mathrm{pH} 9.0$, Tris base $12.1 \mathrm{~g}$, EDTA $3.7 \mathrm{~g}$, distilled water $500 \mathrm{ml}$, boiling) for $2 \mathrm{~min}$. Sections were incubated with affinity purified anti-IL2RB (final concentration $0.5 \mathrm{mg} / \mathrm{ml}$ ) in PBS at $4{ }^{\circ} \mathrm{C}$ overnight and for $1 \mathrm{~h}$ at room temperature and then with biotinylated goat anti-rabbit IgG (OriGene Technologies, Inc., Beijing, China) for $30 \mathrm{~min}$ at $37^{\circ} \mathrm{C}$. Between each step, the slides were washed in PBS (twice, 5 min each wash). The secondary antibody was visualized using DAB reagent (ZSGB-BIO. Inc. Beijing, China). The specimens were then rinsed in distilled water, air dried, mounted with Neutral balsam (Shanghai Yiyang Instrument CO., LTD., Shanghai, China) and observed under Leica DM1000 LED microscope (Leica, Germany).

\section{The fish of miR-216b in the small intestine tissue}

Oar-miR-216b detection probe, scrambled probe and the G-type miRNA in situ hybridization assay kit were purchased from Focobio Corporation (Guangzhou, China). The sections were prepared like IHC and dewaxed in xylenes I, II and III for 5 min each. Then the slides were put in $100 \%, 85 \%, 70 \%$ ethanol for 5 min each. After washed once with PBS for $5 \mathrm{~min}$, and the slides were incubated in solution $\mathrm{A}$ for 20 minutes at room temperature, then the solution A was removed and treated with solution B at room temperature for $15 \mathrm{~min}$. After that, the slides were washed in PBS for $5 \mathrm{~min}$ and fixed in 4\% formaldehyde in PBS for $15 \mathrm{~min}$. The slides were washed in PBS for 10 minutes before addition of $200 \mu \mathrm{l}$ of $\mathrm{C}$ solution at $37^{\circ} \mathrm{C}$ for prehybridization. The $\mathrm{C}$ solution was removed, $10 \mu \mathrm{l}$ of a hybridization solution containing 1.5-2.0 $\mu \mathrm{M}$ mRR-216b probe was added and the slides were crossed overnight in an incubator at $40-42{ }^{\circ} \mathrm{C}$. On the next day, the probe was removed, the slide was washed washing 6 buffer I for 15 min at room temperature, and then rinsed twice in wash buffer II for 15 minutes each time. The slides were washed in $75 \%, 100 \%$ ethanol for $2 \mathrm{~min}$, air dried for $10 \mathrm{~min}$, and then $10 \mu \mathrm{l}$ of DAPI was added for $10 \mathrm{~min}$. The slides could be analyzed by Leica DMI8 fluorescence microscope (Leica, Germany).

\section{miRNA target prediction}

To predict the targets of miR-216b during the process of CE, miRNA-IL2RB pairs were selected using algorithms from RNAhybrid (https://bibiserv.cebitec.unibielefeld.de/Rnahybrid).

\section{Plasmid construction and luciferase reporter assay}

The dual-luciferase reporter vectors construct was generated by cloning the total 3'UTR or the mutant3'UTR of IL2RB into pmiR-GLO vector (Promega, Madison, USA) at the site of NheI and XbaI enzyme digestion. The IL2RB 3'UTR fragment was cloned using PCR. Based on the predicted interaction sites, miR-216b had 8 seed bases paired with IL2RB 3'UTR. Eight of them were mutated by PCR and enzymatic digestion to construct Mut-IL2RB3'UTR. The Firefly luciferase vector as internal reference. The mutated 3'UTR of IL2RB (Mut-IL2RB) was constructed as a negative control. Fifty ng of pmirGLOIL2RB 3'UTR (IL2RB-W) or pmirGLO-Mut-IL2RB 
Table 1: PCR primers.

\begin{tabular}{lllll}
\hline Gene & Forward primer & Reverse primer & Annealing temp $\left({ }^{\circ} \mathrm{C}\right)$ & Product Size (bp) \\
\hline IL2RB & GCACAGAGGAGCTTAATG & TGAGGAGACTCGGAGAGA & 53 & 144 \\
Mut-IL2RB & GCACAGAGGAGCGATTGA & TGAGGAGACTCGGAGAGA & 53 & 147 \\
\hline
\end{tabular}

3'UTR (IL2RB-M) and miR-216b mimics or miR-216b inhibitor were co-transfected into $293 \mathrm{~T}$ cells in a 6-well plate using Lipofectamine 2000 (Invitrogen). After $48 \mathrm{~h}$, all the target validation assays were performed with the dual-luciferase reporter system (Vigorous Biotechnology, Beijing) according to the manufacturer's instructions (Yang et al., 2015). The activity was measured by the BHP9504 Optical Analysis Reader (Hamamatsu, Japan). The fluorescence signal was collected each $0.5^{\circ} \mathrm{C}$ for $20 \mathrm{~s}$. The double $\Delta \mathrm{Ct}$ method was used to measure the expression alteration.

\section{$q R T-P C R$}

Total RNA was extracted with RNA simple Total RNA kit (Tiangen, China) from 293t cells. Single strand cDNAs were prepared from $1 \mu \mathrm{g}$ RNA using a reverse transcription Kit (Takara). The qRT-PCR reactions were set up in 20 $\mu \mathrm{l}$ reaction mixtures containing $10 \mu \mathrm{l}$ lightCycle ${ }^{\circledR} 480$ SYBR Green I Master (Roche), $0.4 \mu$ l sense primers, 0.4 $\mu \mathrm{l}$ antisense primers, $7.2 \mu \mathrm{l}$ distilled water, $2 \mu \mathrm{l}$ template. Reaction conditions were as follows: $95^{\circ} \mathrm{C}$ for $5 \mathrm{~min}$, and then 35 cycles $95^{\circ} \mathrm{C}$ for $10 \mathrm{~s}, 53^{\circ} \mathrm{C}$ for $30 \mathrm{~s}$, and $72^{\circ} \mathrm{C}$ for $30 \mathrm{~s}$. The qRT-PCR primers are listed in Table I.

\section{Statistical analysis}

All images represent at least three independent experiments. Every qRT-PCR, luciferase reporter assay and cell viability assay were repeated in triplicate. The results are expressed as mean \pm SEM. P-values were analyzed by the two-tailed Student's t-test using GraphPad Prism 5 software (IBM), and $\mathrm{p}<0.05$ was considered signifcant.

\section{RESULTS}

To assess the expression and distribution of IL2RB in the intestinal tissue, we performed immunohistochemistry (IHC) analysis. IHC analysis showed that IL2RB protein was highly expressed in the intestinal mucosa of RG sheep compared to NRG at $8 \mathrm{~h}$ after infection, especially in T cells of the epithelial layer (Fig. 1A). In addition, IL2RB expression intensity at sites with immobilized IL2RB antibodies was significantly higher (Fig. 1A and 1B) and was strongly expressed in the duodenum and jejunum. Interestingly, IL2RB is mainly expressed in the intestinal mucosa of RG (Fig. 1C), as well as increasingly expressed from intestinal mucosa to muscle layer in the duodenum and jejunum in the NRG (Fig. 1D). This disparity suggests that the RG sheep had a stronger resistance compared to the NRG sheep during the early of infection with $E$. granulosus eggs. In the former work of our laboratory, the
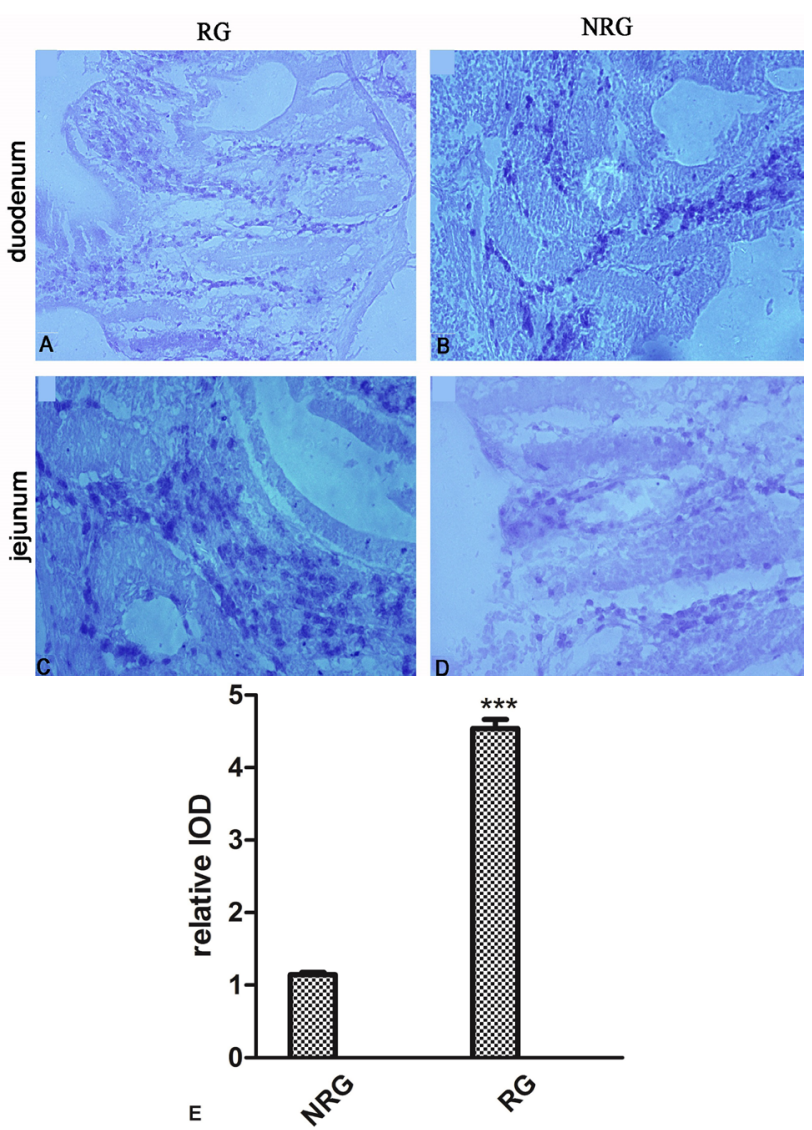

Fig. 1. The distribution of IL2RB in the small intestine of sheep. Intestinal IL2RB expression in NRG and RG sheep was determined by immunohistochemical staining. A, C: Strong IL2RB positive expression was observed in $T$ cells of mucosal layer and musclar layer. B, D: The positive signal of IL2RB was only detected in mucosal layer, especially in the lamina propria and epithelium. E: The average IOD was obtained by analysing IL2RB IHC in four random felds of each slide. Upper panel: representative image; lower panel: quantitative analysis. Results are presented as the mean \pm SEM (magnifcation, $200 \times$, scale bars $=100 \mu \mathrm{m})$. 
IL2RB mRNA level was also upregulated (Hui et al., 2015). These results may indicate some microRNAs regulate the expression level of IL2RB mRNA and protein, and then we performed microRNAs (miR-216b) and targets verification.

Compared with their targets, miRNAs usually show the opposite expression pattern. Therefore, we investigated whether miR-216b was negatively correlated with IL2RB in CE intestinal tissue. Previous study carried out highthroughput sequencing, we found that the expression of miR-216b in the intestinal mucosa of RG CE sheep was significantly lower than that of NRG sheep samples (Fig. 2A). Using miRNA in situ hybridization, we examined miR$216 \mathrm{~b}$ expression in intestinal mucosa and muscular layer T cells (Fig. 2). Animal miRNAs are thought to prevent translation without affecting the level of transcriptions. Our results suggest that IL2RB expression is regulated by a typical miRNA-mediated post-transcriptional mechanism. According to the calculations in active CEs and the inverse correlations of miR-216b and IL2RB protein levels, these data indicate that IL2RB is a miR-216b target.
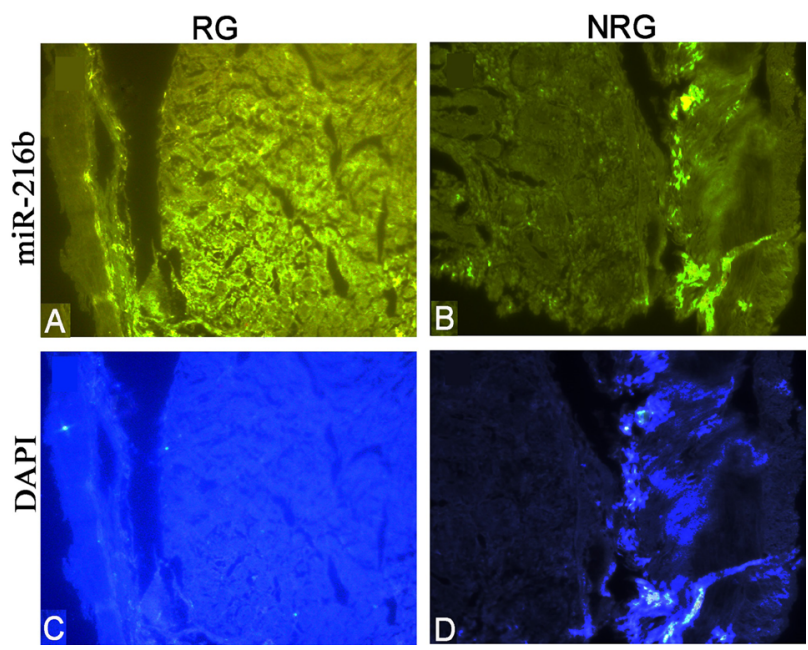

Fig. 2. miR-216b and IL2RB protein levels are inversely correlated in RG and NRG sheep intestinal tissues. miRNA in situ hybridization for miR-216b was performed on intestinal sections from RG and NRG controls (green, miR-216b; blue, DAPI nuclear staining). (Magnifcation $200 \times$, scale bar $=200 \mu \mathrm{m})$.

It is believed that miRNAs regulate the IL2RB expression. The computer-aided algorithms RNAhybrid were used to predict miRNAs that could possibly target IL2RB. Using this approach, seven miRNAs (miR-16a/b, $\mathrm{miR}-15 \mathrm{a} / \mathrm{b}, \mathrm{miR}-216 \mathrm{~b}, \mathrm{miR}-424$, miR-497) indentied that could possibly bind to the sequences of IL2RB 3'-UTR.We observed miR-216b-IL2RB 3'UTR hybrid; the minimum free energy $(\Delta G)$ of miR-216b-IL2RB hybridization was $-21.5 \mathrm{kcal} / \mathrm{mol}$, as shown in Figure $3 \mathrm{~A}$. In addition, there was a perfect base pairing between the seed region and the homologous target. Moreover, the binding sequence of miR-216b in IL2RB 3'-UTR is highly conserved among species (Fig. 3B). Thus, sheep IL2RB may be a direct miR-216b target. We examined the association between miR-216b and IL2RB by altering the level of miR-216b and examining the effect of IL2RB expression on $293 \mathrm{~T}$ cells. We first mimic miR-216b precursors by pre-miR216 b transfection $293 \mathrm{~T}$ cells to overexpress miR-216b, a synthetic RNA oligonucleotide duplex.
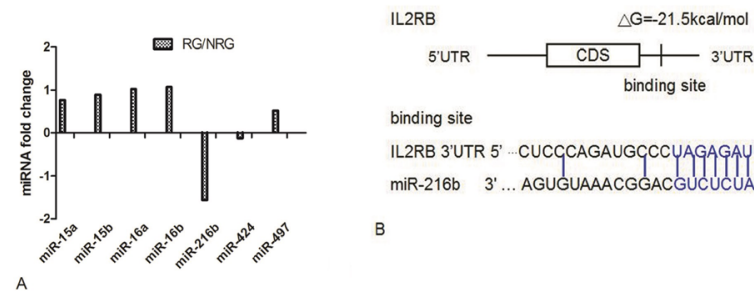

\section{binding site}

IL2RB 3'UTR 5' ... CUCCCAGAUGGCCUAGAGAUUG .... 3' miR-216b $3^{\prime} \ldots$ AGUGUAAACGGACGUCUCUAAA ... $5^{\prime}$

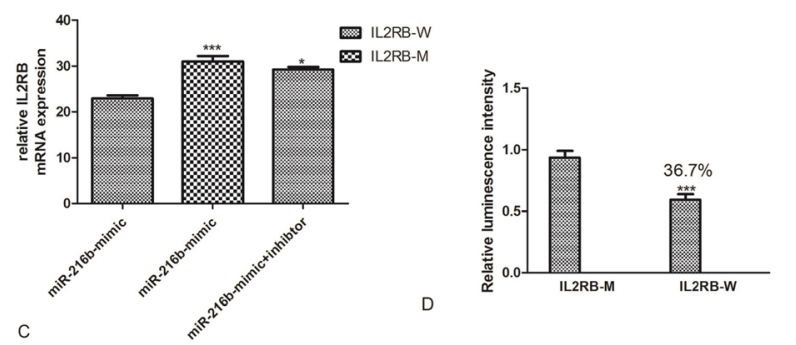

Fig. 3. The IL2RB 3'-UTR contains conserved miR-216b target sites. A: The fold-changes of seven miRNAs predicted by three algorithms in RG intestinal tissues compared to NRG (control), as analysed by high-throughput sequencing. B: Schematic illustration of conserved duplexes formed by IL2RB 3'UTR and miR-216b interactions. The predicted free energy of the hybrid is noted. Paired bases are marked by a blue line. The complementary seed sites are marked in blue. C: Quantitative real-time PCR analysis of IL2RB expression in $293 \mathrm{~T}$ cells transfected with miR-216b mimics, miR-216b inhibitor, IL2RB-W or IL2RB-M. Data are presented as the mean \pm SEM from three independent experiments (293T group: ${ }^{* * *} p=0.000092$, IL2RB-M $v s$. IL2RB-W). D: Relative luminescence intensity detected by a Hamamatsu optical analyze reader after miR-216b mimics and dual-luciferase vectors were co-transfected into 293 T cells $(* * * \mathrm{P}<0.01)$.

It was validated that they did have interaction analyzed by luciferase reporter assay. To further investigate the effects of miR-216b on 293 T cells, negative control small RNAs, miR-216b mimic, miR-216b inhibitor, and in combination 
with miR-216b mimic and its inhibitor were transfected into $293 \mathrm{~T}$ cells, qRT-PCR results manifested that miR$216 \mathrm{~b}$ were transfected efficiently into $293 \mathrm{~T}$ cells (Fig. $3 \mathrm{C})$. Each reporter construct was separately co-transfected with the miR-216b mimics into $293 \mathrm{~T}$ cells. Compared to the mut-IL2RB- 3'UTR control, the luciferase activity declined by about $36.7 \%$ after transfection with miR-216b mimics and IL2RB-3'UTR reporter vector (Fig. 3D). The luciferase analysis showed that ectopic over-expression of miR-216b reduced IL2RB expression via directly binding to IL2RB 3'UTR, indicating that IL2RB is one target of miR-216b.

It was demonstrated that they did analyze the interaction by luciferase reporter assay. In order to further study, the effect of miR-216b on 293T cells, miR216b mimic, miR-216b inhibitor, negative control small RNA were transfected into 293T cells, qRT-PCR results indicated that miR-216b was efficiently transfected into 293 T cells (Fig. 3C). Each reporter construct was co-transfected into $293 \mathrm{~T}$ cells with miR-216b mimics, respectively. Compared to the mut-IL2RB-3'UTR control, the luciferase activity was reduced by about $36.7 \%$ after miR-216b mimic and IL2RB-3'UTR reporter vectors were transfected (Fig. 3D). Luciferase analysis suggested that overexpression of miR-216b reduced IL2RB expression by direct binding to IL2RB 3'UTR, manifested that IL2RB is a target of miR-216b.

\section{DISCUSSION}

In the present study, we demonstrated a miR-216b binding site in the sheep IL2RB mRNA 3'UTR. We measured miR-216b and IL2RB expression levels in sheep pathological intestinal tissue and normal intestinal tissue and found an opposite correlation between the miR$216 \mathrm{~b}$ level and IL2RB mRNA level. This correlation was also demonstrated by luciferase reporter assay, the result showed direct binding between miR-216b and the IL2RB 3'-UTR. We further confirmed that miR-216b directly inhibited IL2RB post-transcriptional expression by overexpressing or inhibition miR-216b in 293 cells. The results showed a clear opposite relationship between the cellular miR-216b level and IL2RB mRNA level in this cell lines. We also demonstrated that miR-216b was located in both intestinal mucosa and muscle layer in intestine tissue by miRNA ISH in RG, compared to the NRG, only in mucosa. Together, these results indicate that $\mathrm{miR}-216 \mathrm{~b}$ is a negative regulator of IL2RB expression in intestinal tissue.

However, there are many problems that cannot be ignored in the prevention and clinical treatment of hydatid disease in today's world. In the field of prevention and control of hydatid disease, there are problems such as the control of transmission source, difficult to cut off the transmission route, making the prevalence of hydatid disease is difficult to cure at the source. In the field of clinical diagnosis and treatment, the diagnosis of hydatid disease mainly rely on B ultrasound, CT and MRI, and PET-CT, imaging diagnosis there is a certain lag. And immunization kit in the early diagnosis of specificity and sensitivity is more difficult. On the other hand, the pathogenesis of hydatid disease has not been recognized, to the early diagnosis and clinical treatment of hydatidosis brought many uncertain factors. Although evidence from sheep models has proved that the parasite infection caused liver and lung damage and the intestinal immune response (Jiang et al., 2016). The intestinal cell immune response (such as T or B cells) is considered an important component of the mucosal immune system. In this study, we reported a direct and important role for miR-216b in regulating IL2RB expression in the early of CE. miRNAs are related to the innate and adaptive immune system and have made significant efforts to determine the expression of miRNAs in autoimmune diseases and their role in the diagnosis and treatment of some diseases, including CE. Nevertheless, most of CE studies focused primarily on the late stages of $\mathrm{CE}$ infection, and the early stage of $\mathrm{CE}$ was not clear.

IL2RB-related miRNAs (miR-15a/b, miR-16a/b, miR-424 and miR-497) are upregulated in intestine tissues in the sheep CE infection model (Yang et al., 2015). Li et al. (2013) reported that miR-216b negatively regulates KRAS in nasopharyngeal carcinoma, and our study demonstrated its role in intestine tissue of sheep.

Indeed, $\mathrm{T}$ activation contributes to the expression of IL2RB during parasite infection (Satoh et al., 2002), and then promote the secretion of IL-2, suggesting that IL2RB may be a useful target for CE clinical monitoring and early mucosal response. Our results also demonstrate that IL2RB expression is upregulated in intestinal mucosa and muscle layer of $\mathrm{CE}$, which is consistent with previous reports.

Furthermore, the expression of IL2RB was inhibited in $293 \mathrm{~T}$ cells after treatment with pre-miR-216b. Together, these results suggest that miR-216b may decrease T cells activation, due to miR-216b's targeted regulation of IL2RB. In short, miR-216b downregulation and IL2RB protein upregulation may play a role in molecular signature of CE. It is worth note that miR-216b may have additional function, prevention parasite eggs invading animal.

The results of miRNA from in situ hybridization shows that miR-216b is expressed in both IECs and T cells in intestinal tissue; thus, both miR-216b-IL2RB signals may be important for CE development. In our 
results indicated that miR-216b as a suppressor of $\mathrm{T}$ cells activation in active CE, the association between miR-216b and $\mathrm{T}$ cells requires further investigation.

\section{ACKNOWLEDGMENTS}

This study was funded by the National Natural Science Foundation of China (Grant No. 3106029). The authors declared that they have no conflicts of interest to this work. We declare that we do not have any commercial or associative interest that represents a conflict of interest in connection with the work submitted.

Statement of conflict of interest

The authors have declared no conflict of interest.

\section{REFERENCES}

Bartel, D.P., 2004. MicroRNAs: Genomics, biogenesis, mechanism and function. Cell, 116: 281-297. https://doi.org/10.1016/S0092-8674(04)00045-5

Chen, F., Liu, Z., Wu, W. and Rozo, C., 2012. An essential role for TH2-type responses in limiting acute tissue damage during experimental helminth infection. Nat. Med., 18: 260-266. https://doi. org/10.1038/nm.2628

Chiang, K., Sung, T.L. and Rice, A.P., 2012. Regulation of cyclin T1 and HIV-1 replication by microRNAs in resting CD4+ T lymphocytes. J. Virol., 86: 32443252. https://doi.org/10.1128/JVI.05065-11

Fan, W., Liang, D., Tang, Y., Qu, B., Cui, H. and Luo, X., 2012. Identification of microRNA-31 as a novel regulator contributing to impaired IL-2 production in $\mathrm{T}$ cells from patients with systemic lupus erythematosus. Arthritis. Rheumaatol., 64: 37153725. https://doi.org/10.1002/art.34596

Glasmacher, E., Kai, P.H., Vogel, K.U., Rath, N., Du, L. and Wolf, C., 2010. Roquin binds inducible costimulator mRNA and effectors of mRNA decay to induce microRNA-independent posttranscriptional repression. Nat. Immunol., 11: 725 733. https://doi.org/10.1038/ni.1902

Hui, W., Jiang, S., Tang, J., Hou, H., Chen, S. and Jia, B., 2015. An immediate innate immune response occurred in the early stage of E. granulosus eggs infection in sheep: Evidence from microarray analysis. PLoS One, 10: e0135096. https://doi. org/10.1371/journal.pone.0135096

Jiang, S., Li, X., Wang, X., Ban, Q., Hui, W. and Jia, B., 2016. MicroRNA profiling of the intestinal tissue of Kazakh sheep after experimental Echinococcus granulosus infection, using a high-throughput approach. Parasite, 23: 23-31. https://doi. org/10.1051/parasite/2016023

Jindra, P.T., Bagley, J., Godwin, J.G. and Iacomini, J., 2010. Costimulation dependent expression of miR214 increases the ability of T cells to proliferate by targeting Pten. J. Immunol., 185: 990-997. https:// doi.org/10.4049/jimmunol.1000793

Kim, S.Y., Lee, Y.H. and Bae, Y.S., 2012. MiR-186, miR216b. miR-337-3p, and miR-760 cooperatively induce cellular senescence by targeting $\alpha$ subunit of protein kinase CKII in human colorectal cancer cells. Biochem. biophys. Res. Commun., 429: 173179. https://doi.org/10.1016/j.bbrc.2012.10.117

Larrieu, E., Herrero, E., Mujica, G., Labanchi, J.L., Araya, D. and Grizmado, C., 2013. Pilot field trial of the EG95 vaccine against ovine cystic echinococcosis in Rio Negro, Argentina: Early impact and preliminary data. Acta Trop., 127: 143-151. https:// doi.org/10.1016/j.actatropica.2013.04.009

Li, M., Yu, M., Liu, C., Zhu, H., He, X., Peng, S. and Hua, J., 2013. miR-34c works downstream of p53 leading to dairy goat male germline stem-cell (mGSCs) apoptosis. Cell Prolif., 46: 223-231. https://doi.org/10.1111/cpr.12013

Magambo, J.K., Wachira, T.M., Wachira, J. and Raasen, T., 1995. Cellular immunity to Echinococcus granulosus cysts. Afr. J. Hlth. Sci., 2: 250-253.

Satoh, M., Toma, H., Sugahara, K., Etoh, K., Shiroma, Y. and Kiyuna, S., 2002. Involvement of IL-2/IL-2R system activation by parasite antigen in polyclonal expansion of CD4+25+ HTLV-1-infected T-cells in human carriers of both HTLV-1 and S. stercoralis. Oncogene, 21: 2466-2475. https://doi.org/10.1038/ sj.onc.1205329

Veit, P., Bilger, B., Schad, V., Schäfer, J., Frank, W. and Lucius, R., 1995. Influence of environmental factors on the infectivity of Echinococcus multilocularis eggs. Parasitology, 110: 79-86. https://doi.org/10.1017/S0031182000081075

Weitzel, R.P., Lesniewski, M.L., Haviernik, P., Haviernik, P., Kadereit, S., Leahy, P., Greco, N.J. and Laughlin, M.J., 2009. microRNA 184 regulates expression of NFAT1 in umbilical cord blood CD4+ T cells. Blood, 113: 6648-6657. https://doi. org/10.1182/blood-2008-09-181156

Yang, Y., Zhou, Q.J., Chen, X.Q., Yan, B.L., Guo, X.L., Zhang, H.L. and Du, A.F., 2015. Profiling of differentially expressed genes in sheep $T$ lymphocytes response to an artificial primary Haemonchus contortus infection. Parasite Vector, 8: 235-247. https://doi.org/10.1186/s13071-015- 
0844-z

Yu, D., Tan, A.H., Hu, X., Athanasopoulos, V., Simpson, N., Silva, D.G. and Goodnow, C.C., 2007. Roquin represses autoimmunity by limiting inducible T-cell co-stimulator messenger RNA. Nature, 450: 299-303. https://doi.org/10.1038/nature06253
Zhu, M., Gao, F., Li, Z., Wang, X., Wang, H., Wang, Z. and Zhao, W., 2016. Immunoprotection of recombinant Eg. myophilin against Echinococcus granulosus infection in sheep. Exp. Ther. Med., 12: 1585-1590. https://doi.org/10.3892/etm.2016.3501 